\title{
E2HRL: An Energy-efficient Hardware Accelerator for Hierarchical Deep Reinforcement Learning
}

\author{
AIDIN SHIRI, UTTEJ KALLAKURI, HASIB-AL RASHID, and BHARAT PRAKASH, University \\ of Maryland Baltimore County, USA \\ NICHOLAS R. WAYTOWICH, Army Research Laboratory, USA \\ TIM OATES and TINOOSH MOHSENIN, University of Maryland Baltimore County, USA
}

\begin{abstract}
Recently, Reinforcement Learning (RL) has shown great performance in solving sequential decision-making and control in dynamic environment problems. Despite its achievements, deploying Deep Neural Network (DNN)-based RL is expensive in terms of time and power due to the large number of episodes required to train agents with high dimensional image representations. Additionally, at the interference the large energy footprint of deep neural networks can be a major drawback. Embedded edge devices as the main platform for deploying RL applications are intrinsically resource-constrained and deploying deep neural network-based $\mathrm{RL}$ on them is a challenging task. As a result, reducing the number of actions taken by the RL agent to learn desired policy, along with the energy-efficient deployment of RL, is crucial. In this article, we propose Energy Efficient Hierarchical Reinforcement Learning (E2HRL), which is a scalable hardware architecture for RL applications. E2HRL utilizes a cross-layer design methodology for achieving better energy efficiency, smaller model size, higher accuracy, and system integration at the software and hardware layers. Our proposed model for RL agent is designed based on the learning hierarchical policies, which makes the network architecture more efficient for implementation on mobile devices. We evaluated our model in three different RL environments with different level of complexity. Simulation results with our analysis illustrate that hierarchical policy learning with several levels of control improves RL agents training efficiency and the agent learns the desired policy faster compared to a non-hierarchical model. This improvement is specifically more observable as the environment or the task becomes more complex with multiple objective subgoals. We tested our model with different hyperparameters to achieve the maximum reward by the RL agent while minimizing the model size, parameters, and required number of operations. E2HRL model enables efficient deployment of RL agent on resource-constraint-embedded devices with the proposed custom hardware architecture that is scalable and fully parameterized with respect to the number of input channels, filter size, and depth. The number of processing engines $(\mathrm{PE})$ in the proposed hardware can vary between 1 to 8 , which provides the flexibility of tradeoff of different factors such as latency, throughput, power, and energy efficiency. By performing a systematic hardware parameter analysis and design space exploration, we implemented the most energyefficient hardware architectures of E2HRL on Xilinx Artix-7 FPGA and NVIDIA Jetson TX2. Comparing the implementation results shows Jetson TX2 boards achieve $0.1 \sim 1.3 \mathrm{GOP} / \mathrm{S} / \mathrm{W}$ energy efficiency while Artix-7 FPGA achieves $1.1 \sim 11.4 \mathrm{GOP} / \mathrm{S} / \mathrm{W}$, which denotes $8.8 \times \sim 11 \times$ better energy efficiency of E2HRL when model
\end{abstract}

This project was sponsored by the U.S. Army Research Laboratory under Cooperative Agreement Number W911NF-102-0022. The views and conclusions contained in this document are those of the authors and should not be interpreted as representing the official policies, either expressed or implied, of the U.S. Government. The U.S. Government is authorized to reproduce and distribute reprints for Government purposes notwithstanding any copyright notation herein.

Authors' addresses: A. Shiri, U. Kallakuri, H.-A. Rashid, B. Prakash, T. Oates, and T. Mohsenin, University of Maryland Baltimore County; emails: aidin.shiri@umbc.edu, ukalla1@umbc.edu, hrashid1@umbc.edu,bhp1@umbc.edu, oates@ cs.umbc.edu, tinoosh@umbc.edu; N. R. Waytowich, Army Research Laboratory; email: nick.waytowich@gmail.com.

ACM acknowledges that this contribution was authored or co-authored by an employee, contractor, or affiliate of the United States government. As such, the United States government retains a nonexclusive, royalty-free right to publish or reproduce this article, or to allow others to do so, for government purposes only.

(C) 2022 Association for Computing Machinery.

1084-4309/2022/09-ART45 \$15.00

https://doi.org/10.1145/3498327

ACM Transactions on Design Automation of Electronic Systems, Vol. 27, No. 5, Article 45. Pub. date: September 2022. 
is implemented on FPGA. Additionally, compared to similar works our design shows better performance and energy efficiency.

CCS Concepts: • Computer systems organization $\rightarrow$ Architectures; $・$ Computing methodologies $\rightarrow$ Machine learning;

Additional Key Words and Phrases: Reinforcement Learning, CNN, energy efficient hardware accelerator, FPGA, CPU

\section{ACM Reference format:}

Aidin Shiri, Uttej Kallakuri, Hasib-Al Rashid, Bharat Prakash, Nicholas R. Waytowich, Tim Oates, and Tinoosh Mohsenin. 2022. E2HRL: An Energy-efficient Hardware Accelerator for Hierarchical Deep Reinforcement Learning. ACM Trans. Des. Autom. Electron. Syst. 27, 5, Article 45 (September 2022), 19 pages.

https://doi.org/10.1145/3498327

\section{INTRODUCTION}

Reinforcement Learning (RL) is a goal-oriented paradigm of machine learning in which the agent tries to learn a policy to achieve complex tasks by trial-and-error. Reinforcement Learning is used for problems that involve sequential-decision making where the agent needs to take actions in an environment to maximize cumulative future rewards. In Reinforcement Learning, goals are specified using a reward function [36]. Human feedback can be used to specify goals as well, as shown in Reference [9]. Applications such as playing video games by receiving only pixelated image inputs, Robotics and robotic arm manipulation, and self-driving vehicles such as autonomous cars and drone navigation system are areas in which Reinforcement Learning has shown great achievements. Despite demonstrating great performance in tasks that previously were hard to accomplish by conventional machine learning methods in recent years, the previous papers in Reinforcement Learning focused on the algorithm and theoretical aspects, and very few works have considered efficient hardware design for RL [21].

Learning the policy for the RL agent can be a complex and time-consuming task. It is important to train the agent in the fastest and most efficient way, especially when the agents act in a realworld environment where performing actions could be expensive. One scalable way to do this is by breaking down the policy that RL agent needs to learn into multiple levels of hierarchy. In this method, desired policy is divided into individual sub-policies. Two neural networks are trained for performing the desired task-one for selecting the proper subgoal and the other for producing the proper action to perform in the environment. Besides making it easy to specify goals and rewards, hierarchical subgoals can additionally be reused in other similar environments that might have the same subgoals.

Standard RL policies struggle with solving long horizon tasks with sparse rewards. Complex tasks can be broken down into smaller sub-tasks and we can make use of this information to build agents with multiple levels of control. We use this notion to build a reinforcement learning agent architecture and experiment with different parts of the network architecture for efficient deployment of RL agents on the embedded devices. The novelty of this article is proposing an efficient hardware architecture for reinforcement learning based on hierarchical policy learning. So far, most of the previous works have focused on only hardware or software aspect of reinforcement learning.

In this article, we propose E2HRL, a hardware-friendly architecture for Reinforcement Learning, which uses hierarchical policy learning. By employing cross stack design methodology, we have designed a scalable and parameterized hardware for efficient deployment of RL on the embedded 
devices. We performed a Neural Network hyperparameter analysis to find the best tradeoff between accuracy and model size, and a scalable low-power hardware is designed for the optimized model and is implemented both on FPGA and ARM CPU. This article makes the following contributions:

- Propose a hardware-friendly architecture for RL that works based on hierarchical policy learning.

- Perform a Neural Network hyperparameter analysis to find the best tradeoff between accuracy and model size.

- Design a scalable and parameterized hardware for efficient deployment of RL on the embedded devices.

- Implementation of the E2HRL model with different levels of parallelism on Artix7 FPGA and compare them in terms of power consumption, energy efficiency, latency, and utilized resources.

The rest of this article is organized as follows: Related prior work is provided in Section 2. Section 3 explains the background of deep Reinforcement Learning and Hierarchical policy learning, along with the environment setup for our experiments. Section 4 describes the proposed E2HRL system architecture. Section 5 talks about neural network optimization and software experimental results and analysis for energy efficiency. Section 6 describes the E2HRL hardware architecture design and implementation results and comparison with similar works. We conclude in Section 7.

\section{RELATED WORKS}

Despite past efforts and early success on Reinforcement Learning, most of the initial works in the RL domain are limited to simple tasks such as training a specific robot [17], automatic inverted flight control [23], and optimizing dialog policy [34]. These works do not take optimizing the power and performance tradeoffs into account. The advent of deep learning has caused significant progress in RL like other machine learning areas, which resulted in the feasibility of creating powerful autonomous agents that can interact with the environment and learn to perform complex tasks over time with trial-and-error. In recent years, several works that have used RL algorithms have been proposed. Recently, Google Deep Mind announced that the AlphaGo Zero beat the previous champion-defeating AlphaGo 100-0 using an algorithm based only on RL, with no human data, guidance, or any domain knowledge beyond the game rules [33]. Authors in Reference [22] have achieved human-level control in many Atari games with Deep RL. Several works are also proposed that use robotic simulators to train the network with an unlimited amount of data and afterward, transferring the knowledge from the simulator to real-world [26, 38]. Training autonomous robots to navigate to designated locations is another application as well [45]. Recent growth in neural networks and deep learning-based algorithms have been coupled with a significant increase in the model size and networks with a high number of parameters. To address the high computational and storage complexity of neural networks, different computing platforms have been engaged for a diverse range of applications. While most of the current research use software solutions based on general-purpose CPU and GPU platforms to address computational issues, specialized hardware accelerators have demonstrated superior performance in terms of energy efficiency and meeting real-time requirements. Domain-specific accelerators can achieve orders of magnitude improvements in performance per watt compared to general-purpose computers for machine learning applications [7]. Despite great interest and innumerable valuable works in design and implementation of hardware accelerators for different Artificial Intelligence and Machine Learning applications such as image classification[14], Recurrent Neural Network-based Language Models [19], automated tools for deep neural networks implementation with resource and perfor- 


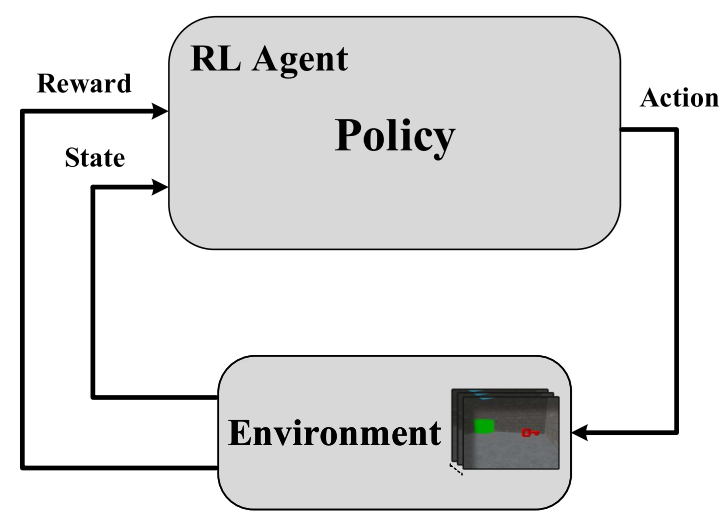

Fig. 1. Traditional Reinforcement Learning agent block diagram. The agent interacts with the environment by executing an action based on its policy. The environment then provides a new state and a scalar reward to the agent.

mance estimation [28, 29], stochastic computing neural network accelerators [20], very few works have considered hardware requirements for RL as well [8,31,32]. Authors in References [15, 16] proposed low-power Deep Reinforcement Learning SoC, which supports CNN, RNN, and FC layers. FPGA accelerators for neural network-based RL has drawn more attention in recent years due to their flexibility and better performance and energy efficiency compared to other heterogeneous platforms $[5,6,30,35,37,40]$. Despite all of the valuable past efforts, still a comprehensive approach for efficient deployment of RL application on hardware design is yet to be proposed. In summary, most of the previous works in the RL domain are limited to the software implementations [24] or on general-purpose GPU at best-case scenarios. Embedded GPUs are among the best devices to handle precise floating-point operations that are able to perform typically at Tera floating-point operations per second (TFLOPS) given a power budget of a few watts (e.g., 1.3 TFLOPS at 7.5 watts for the NVIDIA Jetson TX2 [1]). However, their energy consumption per multiplication or addition operations is still high $(0.17 \mathrm{TOP} / \mathrm{J})$ as a result of handling expensive floating-point operations. Adopting high-precision operations is mandatory for training machine learning algorithms, but during their test and inference, cheaper operations such as binary/ternary operations that consume significantly less energy can deliver the same functionality.

\section{BACKGROUND}

In this section, we present a brief background of reinforcement learning and hierarchical Reinforcement Learning. Then, we describe the environmental setup and how the tasks can be solved using hierarchical Reinforcement Learning.

\subsection{Deep Reinforcement Learning}

Reinforcement Learning agents can learn to solve complex sequential decision making tasks by interacting with the environment, as shown in Figure 1. Typically, we need to define a reward function, state and action spaces, and the actual learning algorithm. Given these ingredients, the agent learns to solve the tasks autonomously without intervention. The agent only gets a reward based on the performance of the tasks to learn the desired policy without specifying how to accomplish the task. Usually, Reinforcement Learning is formalized using Markov Decision Processes (MDP). An MDP is defined as a tuple ( $, A, P, R, \gamma$ ), where $S$ is the state space, $A$ is the action space or the set of actions available to an agent, $\mathrm{P}$ is the unknown transition function, $\mathrm{R}$ is the reward 


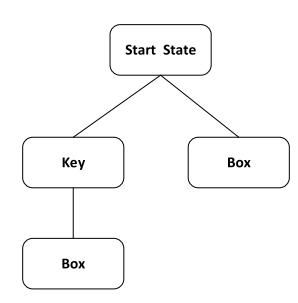

Environment 1: One Level Hierarchy
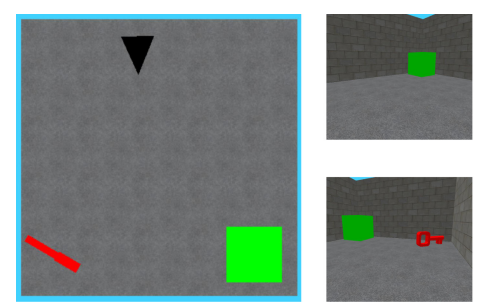

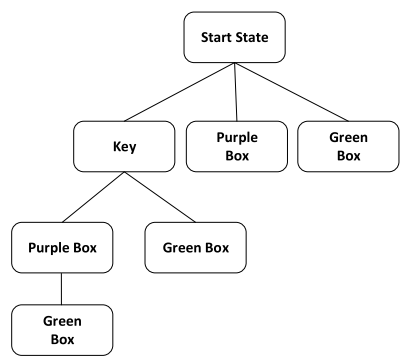

Environment 2: Two Levels Hierarchy
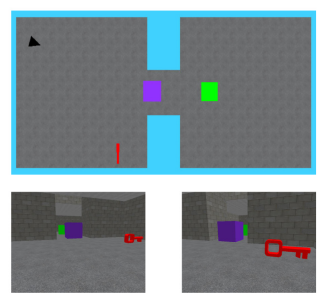

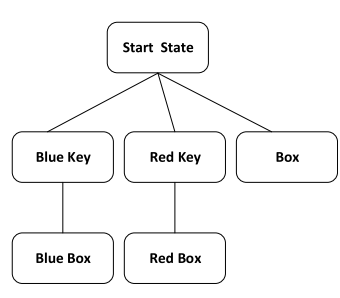

Environment 3: Two Levels Hierarchy

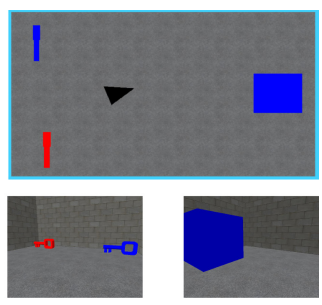

Fig. 2. This figure shows the task graphs for each of these environments in addition to Three MiniWorld environments. In Environment 1, the task is to first collect the red key and then reach the green box. In Environment 2, the task is to first collect the red key and then reach the door, which can be opened only after collecting the key, and then reach the green box (which represents the final goal location). In Environment 3 , The agent has to check the color of the box and then decide to collect the right key and go to the box. The agent receives a reward when it reaches the box, provided it has collected the right key first.

function and $\gamma \epsilon(0,1)$ is the discount factor. The RL agent interacts with the environment by acting according to a policy $\pi$ that is a mapping from states to actions, or a probability distribution over actions. The goal at each step is to maximize the discounted sum of future rewards, $\sum_{t^{\prime}=t}^{\infty} \gamma^{t^{\prime}-t} R_{t^{\prime}}$, and the quality of the policy at time $t$ is measured by the value function $E_{\pi}\left(\sum_{t}^{\infty} \gamma^{t} R_{t+1} \mid s_{0}=s\right)$, with initial state $s$.

\subsection{Hierarchical Reinforcement Learning}

Reinforcement learning techniques have shown great promise in the past few years, getting us a few steps closer to deploying them in the real world. However, there are still many challenges before we can safely and efficiently deploy them in the real world, especially when these technologies work closely with other humans. One of the issues is low sample efficiency, where we require a large number of resources in terms of computing time and data to train these autonomous agents. One way to tackle this problem is to make use of the hierarchical structure present in complex sparse feedback tasks to improve learning. It is possible to use the hierarchical structure and learn multiple levels of control to solve tasks, as shown by References [3] and [18]. These methods have been shown to improve the performance in the sparse reward and long-horizon tasks. In this work, we use the hierarchical structure with two levels of control and in addition with the assumption that we know the subgoals to solve the task. In the following sections, we explain the environmental setup and the E2HRL architecture.

\subsection{Environmental Setup}

Our experiments are performed on the MiniWorld environment [4]. It is a minimalistic 3D environment for Reinforcement Learning and robotics research. The agent can navigate rooms and 
manipulate objects using its first-person view, as shown in Figure 2. In our experiments, we set up three scenarios with different difficulty levels. We assume that humans defining the task have knowledge of how the tasks can be divided into smaller sub-tasks. Sub-tasks are defined prior to training based on domain knowledge. This is similar to some prior work in the field, as seen in Reference [2]. Figure 2 shows more details about how each task is divided into sub-tasks in our experiments.

In Environment 1, shown in Figure 2(a), the agent is in a room that has a box and a key. The agent is spawned at random locations in each episode. The task is to first collect the red key and then reach the green box. The agent receives a reward when it reaches the box, provided it has collected the key first. If the agent reaches the box without the key, then it receives no reward. As described earlier, this task can be decomposed into two subgoals: (1) reach the key, (2) reach the box.

In Environment 2, shown in Figure 2(b), the agent is in a room that has a key. The box is in the other room but is blocked by a purple box (which represents a door). The task is to first collect the red key and then reach the door, which can be opened only after collecting the key. And then reach the green box (which represents the final goal location). The agent receives a reward when it reaches the box, but the only way to reach it is to first collect the key, open the door, and then go to the green exit. If the agent cannot complete the task in a fixed number of steps, then the episode ends and it receives no reward. This task can be decomposed into three subgoals as well: (1) reach the red key, (2) open the door, (3) reach the green exit.

In Environment 3, shown in Figure 2(c), the agent is in a room that has a box, a blue key, and a red key. The agent is spawned at random locations in each episode, and the box is either red or blue. The agent has to check the color of the box and then decide to collect the right key and go to the box. The agent receives a reward when it reaches the box, provided it has collected the right key first. If the agent reaches the box without the key, then it receives no reward. Or, if the agent reaches the box with the wrong colored key, it receives no reward. As described earlier, this task can also be decomposed into two subgoals: (1) reach the red/blue key, (2) reach the box. But here the agent has three subgoals to choose from: (1) reach the red key, (2) reach the blue key, (3) reach the box.

\section{PROPOSED SYSTEM ARCHITECTURE}

In this section, we present an overview of the proposed E2HRL architecture and explain the agent training in detail. E2HRL is designed to perform efficiently as an agent in Reinforcement Learning environments. Figure 5 shows the proposed network architecture of the E2HRL, which consists of convolutional, Dense, LSTM, and softmax layers. We compared standard conventional reinforcement learning baselines with the hierarchical reinforcement learning architecture in terms of accuracy and memory size, as shown in Figure 3. We train both with the same budget in terms of environment steps. As seen in Figure 3, the proposed HRL achieves 7.4× less memory for Environment 1 . For the other two environments, HRL utilizes slightly higher memory, 1.3×, when compared to the baseline. However, the accuracy in terms of final task completion percentage is consistently better for all test cases, 3.7 $\times$ 5.3×. Significant improvement in the task completion rate justifies the slightly higher memory requirement for HRL.

\subsection{E2HRL Architecture}

The agent consists of two modules-the Subgoal module and the Action module. Both these modules get the image embedding from the convolutional layers, which are used to process the image observation. The Subgoal module is responsible for producing the subgoal given the current observation. The action module takes the current observation and the subgoal produced by the sub- 
Task Completion Rate(Accuracy)

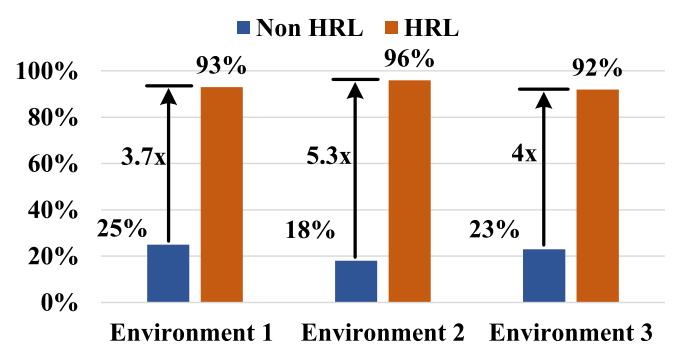

Model Size (KB)

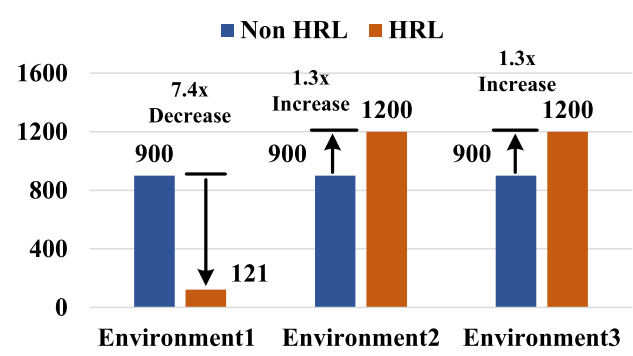

Fig. 3. Comparison between task completion rate and model size of non-hierarchical and hierarchical reinforcement learning models. All models are trained for 1 million steps and their performances are measured afterwards.

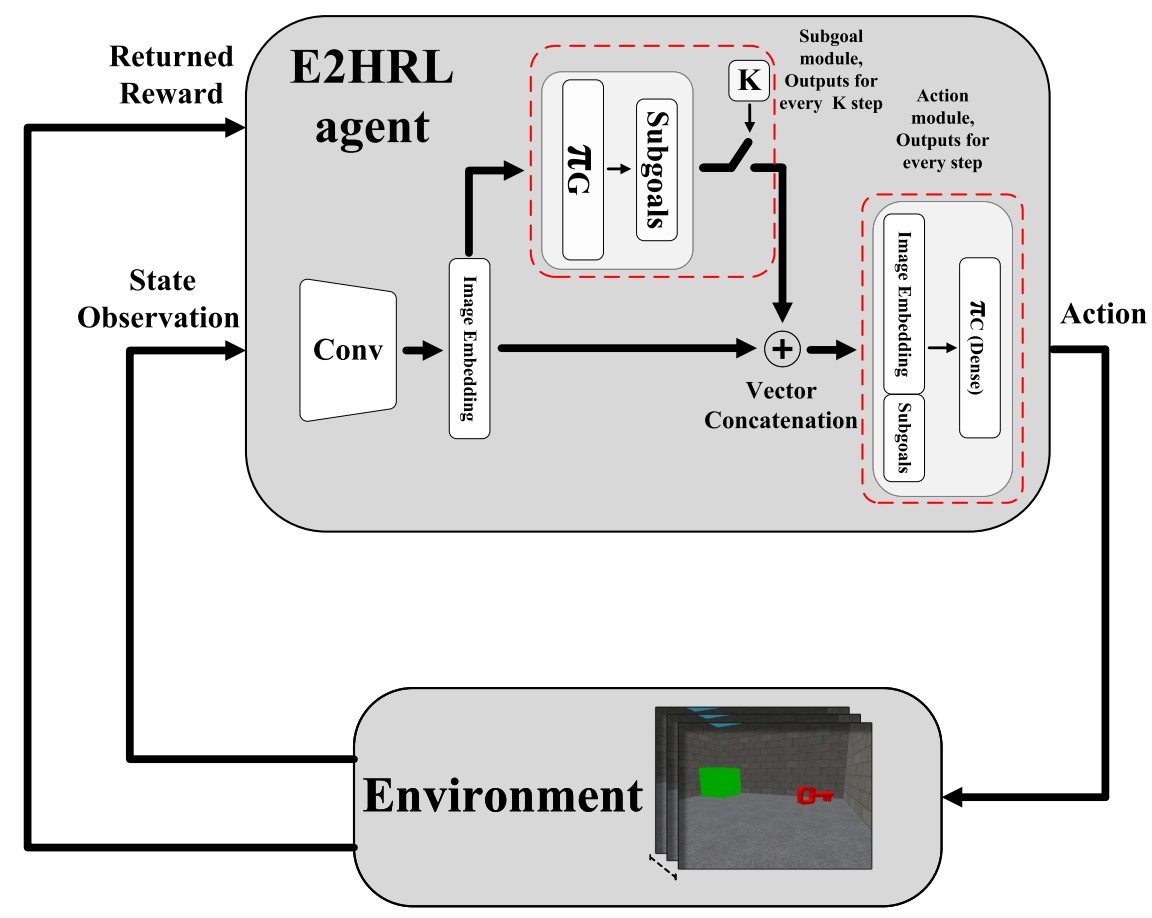

Fig. 4. Block diagram of the proposed architecture of the hierarchical RL agent. Here, the policy $\pi$ of the agent consists of two main modules-the subgoal module $\pi_{G}$ and the action module $\pi_{C}$. The image observation is first processed using a convolution network to get an image embedding. The subgoal module is responsible for providing a subgoal or a high-level action given the current observation. The action module gets the image embedding and the subgoal and calculates the action that is executed in the environment. The subgoal module is activated once every $\mathrm{K}$ steps. The detailed architecture of E2HRL agent is provided in the Figures 4 and 9.

goal module to output the action that is executed in the environment. The architecture is shown in Figure 4, where the agent is trained in two phases. Both policies are trained using proximal policy optimization [27]. The high-level policy does not need to output a goal at every environment timestep. It outputs a subgoal and sleeps for several timesteps $(K)$, which is then used by $\pi_{C}$ 


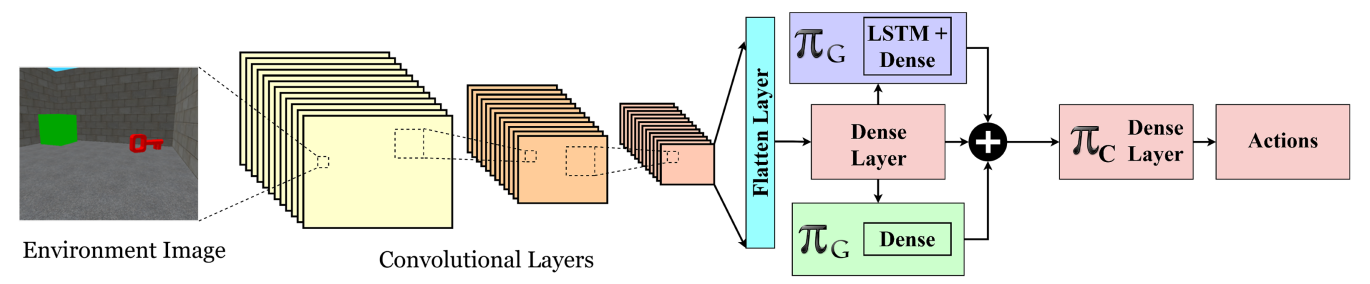

Fig. 5. The proposed network architecture of the E2HRL, as described in Figure 4. The subgoal module has two configurations. One with fully connected (FC) layers shown in green and one with LSTM shown in purple.

to output low-level actions. Additionally, this helps to reduce the hardware and time complexity. We experiment with different intervals, $K$, and reported the results in the next section.

\subsection{Training the Subgoal and Action Modules}

First, the low-level policy $\left(\pi_{C}\right)$ is trained to perform the subgoals. This is done by concatenating a one-hot encoded task ID to the actor network. The agent is trained to perform the subgoals given by these task IDs. Once the agent is able to perform these individual subgoals, the action module is frozen and the subgoal modules are trained in the next phase. In the second phase, the high-level policy $\left(\pi_{G}\right)$ is trained to select subgoals. This output is then concatenated to the low-level policy, which executes a policy to reach the subgoals. As seen in Figure 4, the subgoal module consists of an MLP with multiple fully connected layers. The input to these layers is the image embedding from the convolutional image encoder. This architecture is sufficient for the first simple task. But the second and third tasks are more complicated and need some form of memory.

Therefore, we add an LSTM layer after we get the image encoder and before passing it to the MLP. Long Short-Term Memory (LSTM) is a particular type of recurrent neural network that has internal contextual state cells that act as long-term or short-term memory cells. This is an important characteristic when the prediction of the neural network depends on the historical context of inputs, rather than only the last input. This modification seems to help solve the tasks faster and is required for the second and the third task.

\section{E2HRL NEURAL NETWORK OPTIMIZATION FOR ENERGY EFFICIENCY}

Neural network optimization is the process of systematic model architecture engineering to achieve the best possible performance with the smallest model size and least computation. We performed extensive analysis on the model search space and evaluated our model performance. Search space defines the networks that can be examined to produce the final architecture. The initial E2HRL model consists of three convolution layers, a flatten layer, and a dense (fully connected) layer with an output of 32 for generating an image embedding vector. Next, the embedding vector is fed to the subgoal module, which is a dense or LSTM layer to produce the subgoal vector. Finally, subgoal vector and image embedding are concatenated together to feed another dense layer with Softmax activation and generate the desired output for the agent to act in the environment. The initial HRL agent architecture is shown in detail in Table 1.

We performed a macro-architecture search for layer type, hyper-parameters, and connections with other layers to achieve the best performance. Furthermore, for each block, we optimize the convolutional layer kernel size and filter number, as well as the dropout rate, the existence of a pooling layer after each convolution layer. A number of optimum architectures that produced the best results for the three environments discussed in the previous section are summarized in 
Table 1. FC-HRL and LSTM-HRL Neural Network Architecture in Detail

\begin{tabular}{llcccc}
\hline FC-HRL Layers & LSTM-HRL Layers & Input & Window & \# Kernels & Activation \\
\hline Conv 1 & Conv 1 & $40^{*} 30^{*} 3$ & $(3,3)$ & 32 & ReLU \\
\hline Conv 2 & Conv 2 & $20^{*} 15^{*} 32$ & $(3,3)$ & 32 & ReLU \\
\hline Conv 3 & Conv 3 & $10^{*} 8^{*} 32$ & $(3,3)$ & 32 & ReLU \\
\hline Flatten & Flatten & $5^{*} 4^{*} 32$ & - & - & - \\
\hline Dense $[\pi \mathrm{G}]$ & LSTM $[\pi \mathrm{G}]$ & 32 & - & - & ReLU \\
\hline Dense $[\pi \mathrm{C}]$ & Dense $[\pi \mathrm{C}]$ & 32 & - & - & ReLU \\
\hline Action & Action & 3 & - & - & Softmax \\
\hline
\end{tabular}

Table 2. E2HRL Different Network Configurations with Different Computation and Memory Complexity

\begin{tabular}{lccccccc}
\hline & \multicolumn{3}{c}{ FC-HRL (Environment 1) } & \multicolumn{4}{c}{ LSTM-HRL (Environments 2 \& 3) } \\
& FC1 & FC2 & FC3 & LSTM2 & LSTM4 & LSTM8 & LSTM16 \\
\hline \# Parameters & $11.7 \mathrm{~K}$ & $\mathbf{3 0 . 2} \mathbf{K}$ & $94 \mathrm{~K}$ & $39 \mathrm{~K}$ & $\mathbf{3 9 ~ K}$ & $39 \mathrm{~K}$ & $39 \mathrm{~K}$ \\
\hline Memory & $45.5 \mathrm{~KB}$ & $\mathbf{1 2 1} \mathbf{~ K B}$ & $376 \mathrm{~KB}$ & $1.2 \mathrm{MB}$ & $\mathbf{1 . 2 ~ \mathbf { ~ B }}$ & $1.2 \mathrm{MB}$ & $1.2 \mathrm{MB}$ \\
\hline \# Computations & $2.8 \mathrm{M}$ & $\mathbf{9 . 3} \mathbf{M}$ & $32.3 \mathrm{M}$ & $2.4 \mathrm{M}$ & $\mathbf{2 . 4} \mathbf{M}$ & $2.4 \mathrm{M}$ & $2.4 \mathrm{M}$ \\
\hline Task Completion & $84 \%$ & $\mathbf{9 3 \%}$ & $98 \%$ & $94 \%$ \& $90 \%$ & $\mathbf{9 6 \%} \mathbf{9 2 \%}$ & $96 \%$ \& $92 \%$ & $96 \%$ \& $80 \%$ \\
\hline
\end{tabular}

Each model is trained for one million steps.

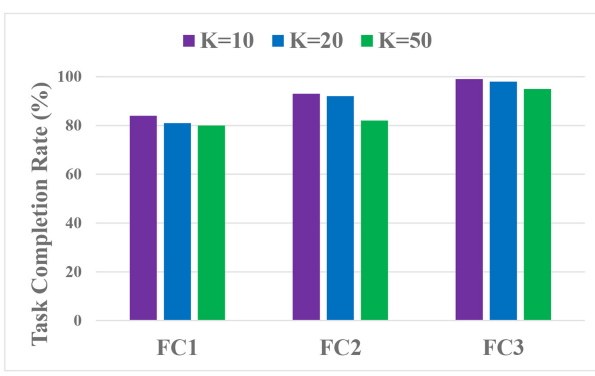

(a)

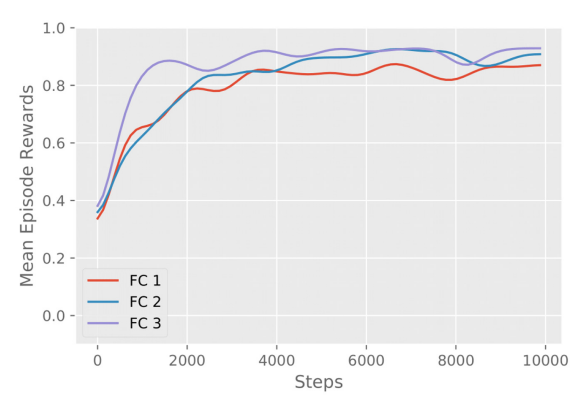

(b)

Fig. 6. Environment 1 (a) Performance and Memory size of hierarchical RL agent for different configurations with respect to K. (b) Reward achieved by the HRL agent in the MiniWorld environment with respect to the number of episodes for different configurations.

Table 2. By comparing the number of parameters and model size of different configurations, we observed that Config 2 for FC-HRL and LSTM4 for LSTM-HRL achieves almost the same reward as the best configurations, while having less memory and computation complexity. Therefore, we select Config 2 and LSTM4 as the optimal design for implementing on hardware. Figures 6, 7, and 8 illustrate the model size and performance of different configurations with respect to the timestep, $K$, amount of timesteps that $\pi_{G}$ sleeps. We observe that for a simple environment like Environment 1 , the model performs better with smaller $\mathrm{K}$. As the complexity of the environment increases, the models perform better when $\mathrm{K}$ is larger.

\section{E2HRL HARDWARE ARCHITECTURE DESIGN, IMPLEMENTATION, AND ANALYSIS}

This section describes the hardware architecture design and implementation of E2HRL with commercial off-the-shelf devices. We have implemented our method on the Xilinx Artix-7 FPGA board 


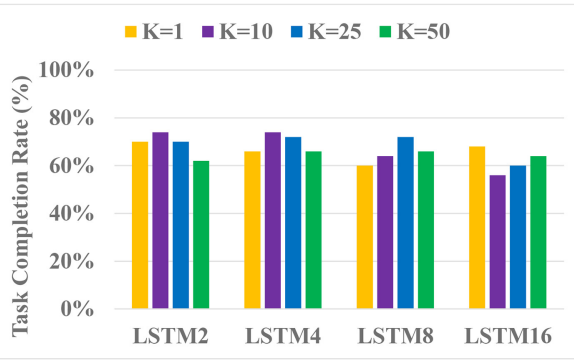

(a)

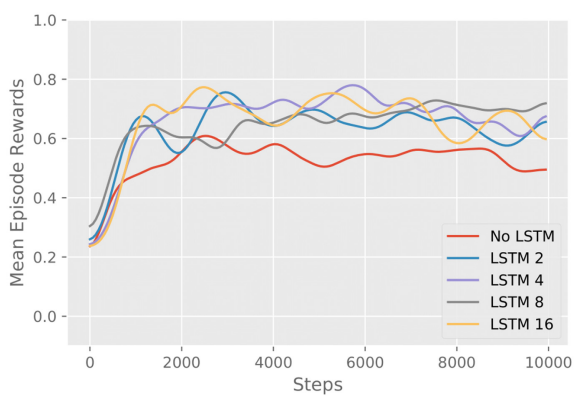

(b)

Fig. 7. Environment 2 (a) Performance and Memory size of hierarchical RL agent for different configurations with respect to K. (b) Reward achieved by the HRL agent in the MiniWorld environment with respect to the number of episodes for different configurations.

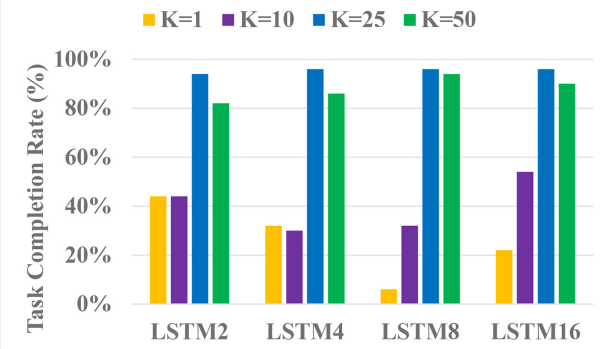

(a)

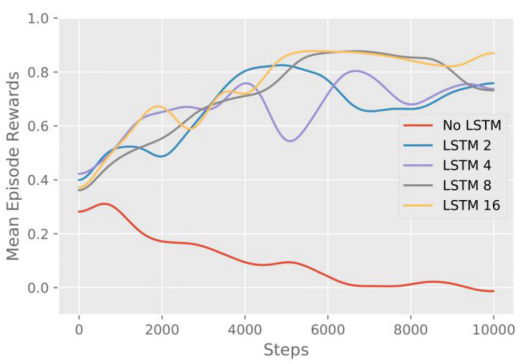

(b)

Fig. 8. Environment 3 (a) Performance and Memory size of hierarchical RL agent for different configurations with respect to K. (b) Reward achieved by the HRL agent in the MiniWorld environment with respect to the number of episodes for different configurations.

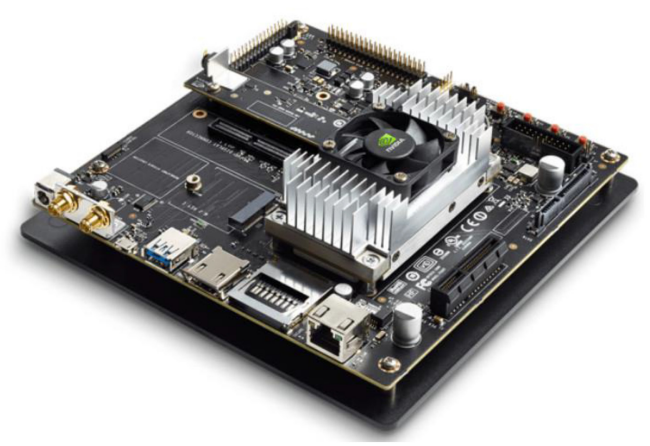

Nvidia Jetson TX2

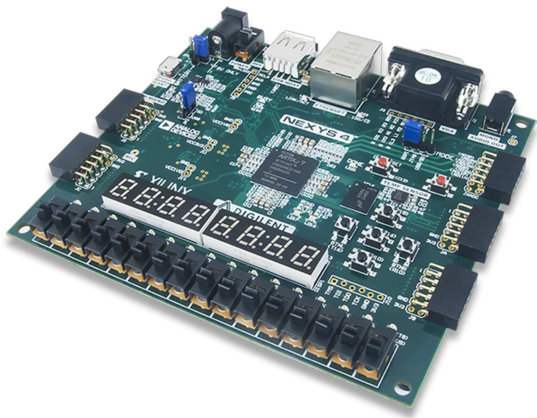

Xilinx Artix-7

Fig. 9. Common off-the-shelf edge platforms.

and NVIDIA Jetson TX2 (shown in Figure 9) to measure the energy-efficient implementations for embedded devices. 


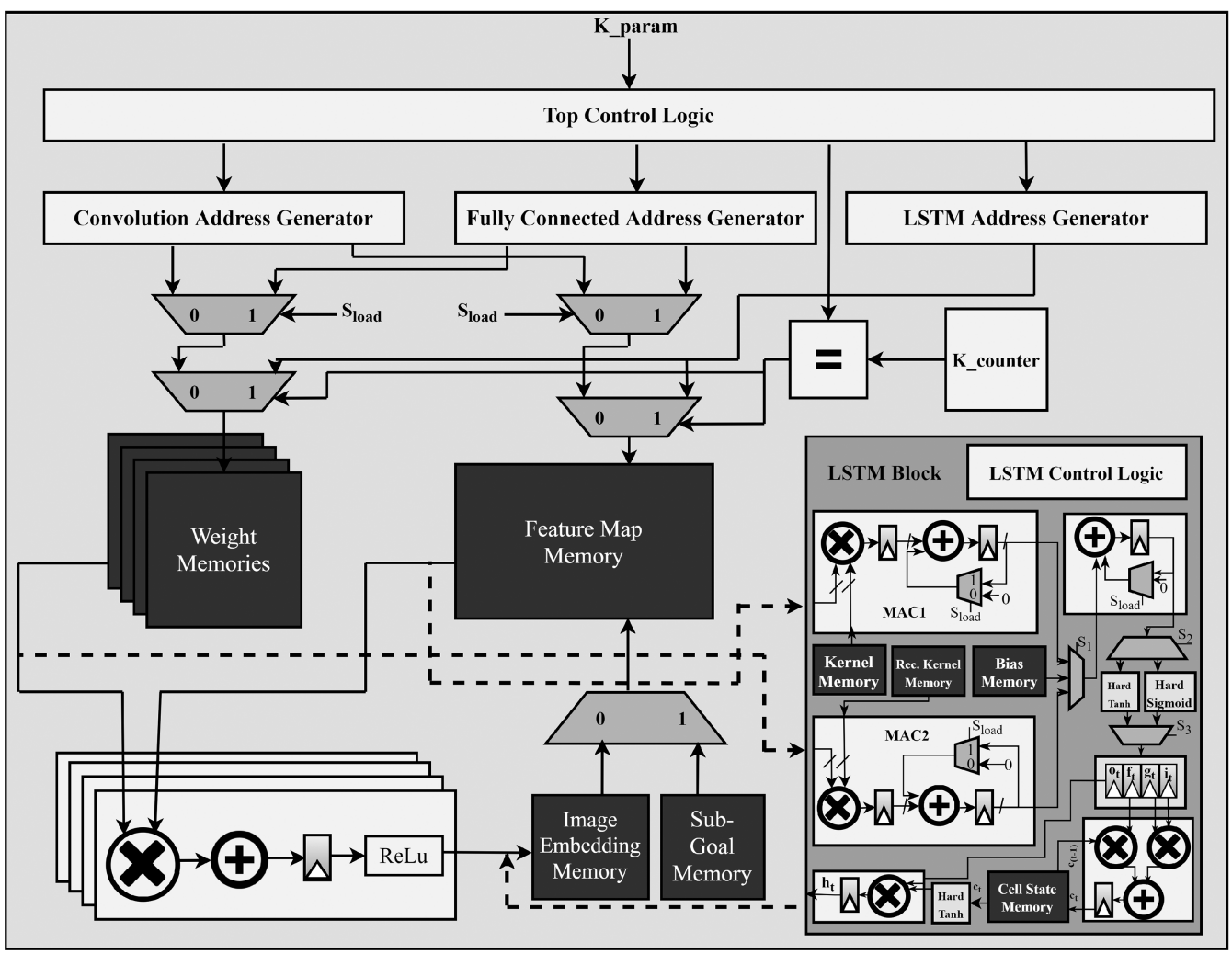

Fig. 10. Block diagram of the proposed E2HRL hardware architecture, which consists of Convolution, LSTM, and Fully Connected layers. As shown in 5, for K-1 number of operations, the design runs through a sequence of Convolutions and Fully Connected layers; for the $K$ th round, the design utilizes an additional Fully Connected layer (in case of FC-HRL) or the LSTM layer (in case of LSTM-HRL).

\subsection{Hardware Architecture Design}

Figure 10 shows the detailed block diagram of the E2HRL hardware architecture. The architecture mainly focuses on important hardware facets such as on-chip memory re-utilization and parallel computations. Another key feature is to fit the hardware on smaller FPGAs with smaller Block RAM sizes. This additionally implies the hardware should occupy minimum area while meeting the latency requirements. The designed hardware is such that it can be easily re-configured and meanwhile portable. The reconfiguration is mainly targeted at implementing newer models, improving the overall performance, and improving the parallelizations. This is achieved by increasing the memory and the MAC widths, changing the memory sizes, and increasing the number of PEs in the design. In Figure 10, the main blocks are the PE array to perform MAC operations in parallel, memory blocks to hold the weight, feature and the intermediate outputs; individual address generation blocks for the convolution and fully connected layers and an independent LSTM block (in the case of LSTM-HRL). The top control logic generates the necessary control signals based on the input configuration. It generates the controls for the convolution, fully connected, and the LSTM address generation blocks as well. The generated addresses are passed on to the available on-chip memories to retrieve the corresponding weight and feature values to be passed into the PE array. The design of the PE has been done in such a way that it can perform one MAC operation per 
clock cycle. The MAC values outputted from the PE arrays are stored in the feature memory as input to the layer that follows after the current computation. The top control logic also accepts a hyper-parameter K. By maintaining an internal k-counter and comparing the two values, it keeps track of when the FC/LSTM branch has to be run. The output of this branch is written in the last section of the feature map, which allows saving the previous FC/LSTM output until it is re-run The architecture for the LSTM block is presented in the Figure 10. The main blocks in the designed hardware include:

- Convolution: The convolution block allows to perform the 2D convolution operations through repeated MAC operations. The control unit generates the necessary control signals to enable the blocks. The convolution address generator generates the addresses according to the tiling scheme explained in Figure 12(c), where a single input channel is selected and convoluted with multiple filters parallelly. Once the MACs have the $\mathrm{N}$ parallel data values from the Weight and the data memories, it performs the MACs in parallel and stores the results sequentially in the Image embedding memory.

- Fully Connected: The operations performed are similar to that of Convolution. The top control logic generates the necessary control signals, and the address generator passes the addresses to the memories, which are fetched into the PE-array. MAC results are stored back into the appropriate memory, depending on the hyper-parameter values.

- LSTM: This is the module on the branch that is executed depending on the hyper-parameter $k$. Simplifying Equations (1)-(4), we can observe that it aims at implementing matrix vector multiplication. Therefore, by the concatenation of the kernel and the recurrent kernel weights into two memories, the memory footprint can be minimized. From Figure 10, we can notice the LSTM module is composed of two MAC modules and memories. The memories hold the kernel, recurrent kernel weights, biases, and cell-state data, respectively. Additionally, the module also performs Hard Tanh and Hard Sigmoid activations.

- Memory: The memory modules on the hardware are mainly divided among the weight, feature, image embedding, and the subgoal memories. For both the designs, i.e., FC-HRL and the LSTM-HRL modules, the input sizes are $40 \times 30 \times 3$. The Weight Memory is used to hold all the weights needed for the convolution, fully connected, and the LSTM layers. The Feature Map memory is designed to keep a maximum intermediate features (i.e., the largest feature map in the convolution layer). The LSTM layer, additionally, has dedicated memories to hold the kernel values, recurrent kernel values, the bias values, and the cell state. Contents from these memories are read into the MAC modules in accordance with the control signals received from the LSTM control logic. Additionally, the top Weight Memory is halved and replicated each time the number of PEs doubles; this allows for convenient data sharing for the tiling scheme.

According to Reference [11], at a particular timestep $t$, the computation inside the LSTM unit is done as per the following equations:

$$
\begin{gathered}
i_{t}=\operatorname{sigmoid}\left(W_{x_{i}} x_{t}+W_{h_{i}} h_{t-1}+b_{i}\right), \\
f_{t}=\operatorname{sigmoid}\left(W_{x_{f}} x_{t}+W_{h_{f}} h_{t-1}+b_{f}\right), \\
o_{t}=\operatorname{sigmoid}\left(W_{x_{o}} x_{t}+W_{h_{o}} h_{t-1}+b_{o}\right), \\
g_{t}=\tanh \left(W_{x_{g}} x_{t}+W_{h_{g}} h_{t-1}+b_{g}\right), \\
c_{t}=f_{t} \bigodot c_{t-1}+i_{t} \odot g_{t}, \\
h_{t}=\tanh \left(c_{t}\right) \bigodot o_{t},
\end{gathered}
$$




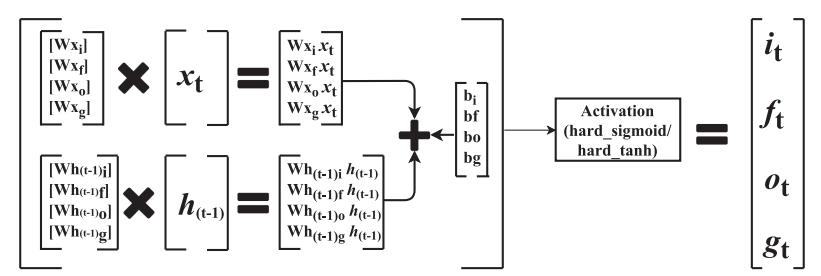

Fig. 11. Concatenation of Equations (1)-(4) for efficient hardware implementation.

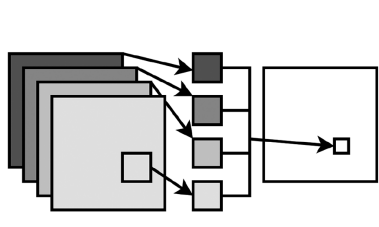

(a)

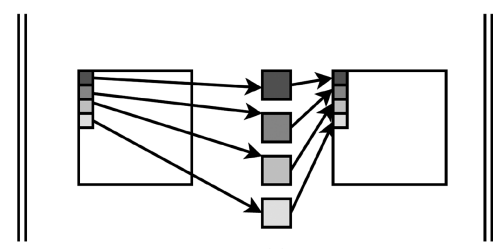

(b)

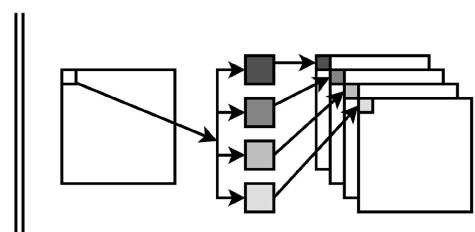

(c)

Fig. 12. (a) Input channel tiling, (b) Image patch tiling, (c) Output channel tiling examination of various parallel tiling procedures for convolutional layers. Output channel tiling has the least memory communication conflict and hence is chosen as tiling scheme for proposed hardware.

where $\odot$ is the element-wise multiplication. $W_{x_{i}}, W_{x_{f}}, W_{x_{o}}, W_{x_{g}}$ are the kernel weights and $W_{h_{i}}$, $W_{h_{f}}, W_{h_{o}}, W_{h_{g}}$ are the recurrent kernel weights, $i_{t}$ is input gate, $f_{t}$ is forget gate, $o_{t}$ is output gate, $g_{t}$ is block input gate, $c_{t}$ is cell state, and $h_{t}$ is LSTM output.

Analyzing Equations (1)-(4), we can conclude that it aims to implement matrix vector multiplication. Hence, by concatenating the kernel and the recurrent kernel weights into two memories, as shown in Figure 11, the memory allocation can be minimized. This allows efficient hardware implementation as well. The LSTM block consists of two MAC modules accompanied by more arithmetic logic and additional memories. The memories hold kernel and recurrent kernel weights, biases, and cell-state data, respectively. It also performs Hard Tanh and Hard Sigmoid activations dynamically.

Originally, LSTM uses sigmoid and tanh as activation functions, as we can see from Equations (1)-(6). As both of these functions are non-linear, hardware implementation is more complex than other activation functions such as ReLU. To reduce the hardware complexity, we have used hard sigmoid and hard tanh defined by the following equations:

$$
\begin{gathered}
\operatorname{hardtanh}(x)=\left\{\begin{array}{lr}
-1, & x<-1 \\
x, & -1 \leq x \leq 1 . \\
1, & x>1
\end{array}\right. \\
\operatorname{hardsigmoid}(x)=\left\{\begin{array}{lr}
0, & x<-2.5 \\
0.2 \times x+0.5, & -2.5 \leq x \leq 2.5 \\
1, & x>2.5
\end{array}\right.
\end{gathered}
$$

Figure 12 shows the basic methods for tiling in convolution. In Figure 12(a) (input channel tiling), a single feature map is convoluted collaterally by multiple input feature map channels. In Figure 12(b) (image patch tiling), the input feature map is divided into smaller sections and is convoluted parallelly. Finally, in Figure 12(c) (output channel tiling), a single input channel is selected and is convoluted with multiple filters parallelly. From Reference [43], we can conclude the best throughput and best parallel memory access and computation is achieved in the output tiling scheme. The Convolution implemented here follows this output tiling scheme. This is achieved by reading $\mathrm{N}$ values from the feature map memory while reading the number of $\mathrm{PE}$ times $\mathrm{N}$ number 
Table 3. The Implementation Results of Proposed E2HRL Hardware on Xilinx Artix 7 FPGA

\begin{tabular}{|c|c|c|c|c|c|c|c|c|c|}
\hline $\begin{array}{l}\text { Model } \\
\text { Config. }\end{array}$ & \#PE & LUT & BRAM & DSPs & $\begin{array}{c}\text { Power } \\
(\mathrm{mW})\end{array}$ & $\begin{array}{c}\text { Latency } \\
(\mathrm{ms})\end{array}$ & $\begin{array}{c}\text { Energy } \\
(\mathrm{mJ})\end{array}$ & $\begin{array}{l}\text { Performance } \\
\text { (GOPS) }\end{array}$ & $\begin{array}{c}\text { Energy Efficiency } \\
\text { (GOPS/W) }\end{array}$ \\
\hline \multirow{4}{*}{$\begin{array}{c}\text { FC } \\
\text { HRL }\end{array}$} & 1 & 1,249 & 52 & 11 & 295 & 5.15 & 1.5 & 0.5 & 1.7 \\
\hline & 2 & 1,797 & 52 & 15 & 308 & 2.67 & 0.9 & 1 & 3 \\
\hline & 4 & 2,236 & 52 & 23 & 322 & 1.47 & 0.5 & 2 & 6.2 \\
\hline & 8 & 3,186 & 52 & 39 & 348 & 0.9 & 0.3 & 4 & 11.4 \\
\hline \multirow{4}{*}{ LSTM HRL } & 1 & 2,485 & 54 & 26 & 336 & 6.4 & 2.1 & 0.4 & 1.1 \\
\hline & 2 & 3,033 & 54 & 30 & 349 & 4.1 & 1.4 & 0.6 & 1.7 \\
\hline & 4 & 3,472 & 54 & 38 & 363 & 2.85 & 1 & 0.8 & 2.2 \\
\hline & 8 & 4,422 & 54 & 54 & 389 & 2.3 & 0.8 & 1 & 3 \\
\hline
\end{tabular}

The results are obtained at a clock frequency of $100 \mathrm{MHz}$, and the required latency to reach target $30 \mathrm{FPS}$ is $33.34 \mathrm{~ms}$.

of Weight values. $\mathrm{N}$ values from the PEs are concatenated and stored as input for the upcoming operation.

We implemented E2HRL hardware on low-power Xilinx Artix-7 FPGA to measure its performance. Hardware is designed to be configurable with different hyper-parameters to meet custom application requirements such as low power consumption or high throughput. Parameters include the number of processing elements (PE), filter shapes, number of filters in the convolutional layers, sizes of the dense layers that are configurable to engage maximum parallel processing ability or utilize the least possible hardware resources. The hardware consists of three main units: (1) Convolution block for performing Convolution operation; (2) Fully Connected block that implements the dense layer functionality or an LSTM block to implement the LSTM operations; (3) On-chip memory that stores image embedding and subgoal vector. The convolution block operates with a multiplier embedded within it. Along with that, there are separate memory blocks for saving weights and feature maps. The input data is fed to the convolution block to calculate the valid convolution of the input using a multiplication unit (MU) and an adder with ReLu activation logic. Strides of two in each direction replaced the time-consuming max-pooling operation. Following the convolutional layers, the first fully connected layer reads the data from the image embedding memory and operates using one multiplier-adder and a few registers. The address flow and control unit generates memory addresses depending on the layer functionality of either convolution, dense or LSTM. Later, the subgoal vector generated by the Fully Connected/LSTM layer is concatenated with the output of the convolution layer and forms the input of the final Fully Connected layer with Softmax activation to produce proper action. The output of this layer is the action generated by the agent to navigate toward the relevant task within the environment. The control unit manages the data flow through different parts of the hardware. 32-bit fixed-point number format is used for representing the data inside the hardware.

\subsection{Xilinx Artix-7 Implementation Results}

As already discussed, the hardware architecture is written in Verilog HDL and is configurable for the desired number of PEs, data, and memory widths. Table 3 shows the FPGA implementation results for the E2HRL hardware on the Artix-7 board obtained from the Xilinx Vivado Design Suite. The results illustrate FPGA utilization, latency, power, energy, and performance for the design while varying the number of PEs from 1 through 8 at $100 \mathrm{MHz}$ operating frequency. The peak performance of the design using its best configuration (i.e., at $100 \mathrm{MHz}$ with $8 \mathrm{PEs}$ ) is 6.4 GOPS. The design with this configuration achieves an average performance of 4 GOPS for the FC-HRL model while achieving 1 GOPS for the LSTM-HRL model. The energy efficiency peaks at 11.4 GOPS/W for the $8 \mathrm{PE}$ design at $100 \mathrm{MHz}$ for the FC-HRL and 3GOPS/W for the LSTM-HRL. 

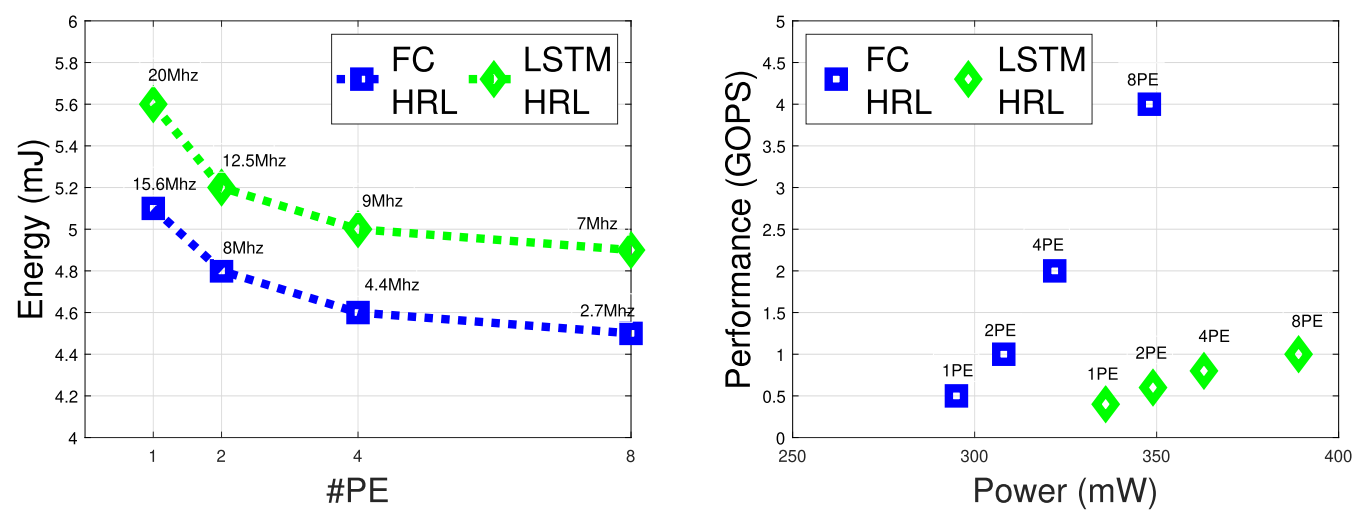

Fig. 13. Impact of the increasing number of PEs on Energy (left) when running the design to meet a deadline of $30 \mathrm{fps}$. Power and performance tradeoff for the FC and LSTM HRL models (right).

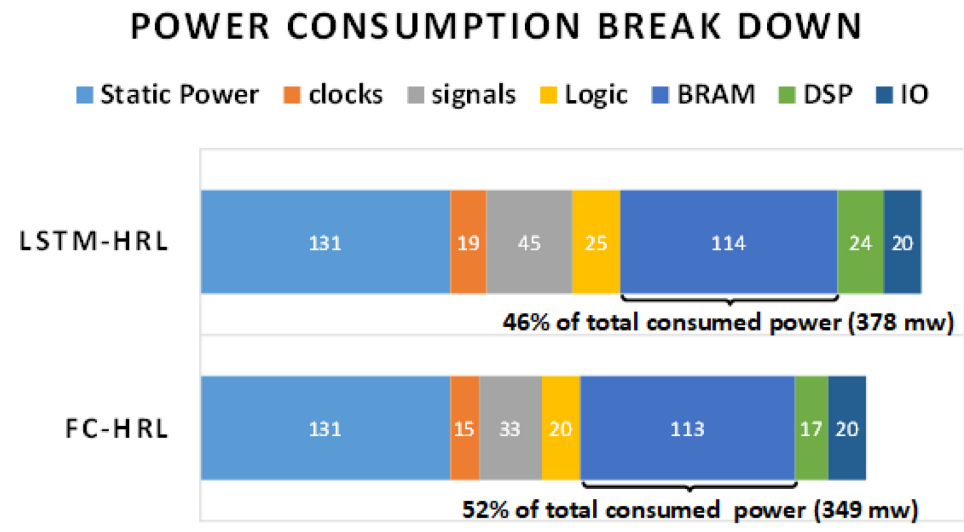

Fig. 14. Power consumption breakdown of the FC-HRL and LSTM HRL hardware architecture on Artix-7 FPGA. The figure shows the breakdown w.r.t. the static and dynamic power consumption. It can be observed that the power consumed by the block-RAMs (BRAMs), 52\% and $46 \%$ for the FC-HRL and the LSTM-HRL models, respectively, are the major portion of the consumed power.

Figure 13 (right) shows the power consumed for increasing performance when the design is running at $100 \mathrm{MHz}$. For the FC-HRL model (blue) the performance reaches $4 \mathrm{GOPS}$ at $8 \mathrm{PE}, 100 \mathrm{MHz}$ while consuming $348 \mathrm{~mW}$ power. Similarly, for the LSTM-HRL (green) the performance reaches 1 GOPS at $8 \mathrm{PE}, 100 \mathrm{MHz}$ while consuming $389 \mathrm{~mW}$ power. The aim of this hardware is to implement it on embedded devices. Hence, we can say that the required deadline to meet is $30 \mathrm{fps}$, the average number of frames an embedded video camera can generate. All configurations in Table 3 meet this deadline of 33.34 ms comfortably. In Figure 13 (left), the impact of increasing the number of PEs on energy when the design is running to meet the deadline of $30 \mathrm{fps}$ is represented. As the number of PEs is increased, the energy drops rapidly initially and gradually saturates. At $8 \mathrm{PE}$ the energy consumed is $4.5 \mathrm{~mJ}$ for the FC-HRL model and $4.9 \mathrm{~mJ}$ for the LSTM-HRL model. Additionally, Figure 14 shows the power usage breakdown for the FC-HRL and the LSTM-HRL models implemented on Artix-7 FPGA. The figure shows the breakdown w.r.t. the static and dynamic power consumption. The breakdown for the dynamic power is represented as well. From the figure, we can observe that the power consumed by the block-RAMs consume $52 \%$ and $46 \%$ for 
Table 4. Deploying the Model to Commercial Off-the-shelf Devices Including a Dual-core Denver CPU, a Quad-core ARM A57 CPU, and a Combination of ARM CPU + Pascal GPU from the NVIDIA TX2 Board

\begin{tabular}{|c|c|c|c|c|c|c|c|}
\hline $\begin{array}{c}\text { Model } \\
\text { Config. }\end{array}$ & $\begin{array}{c}\text { TX2 } \\
\text { Config. }\end{array}$ & $\begin{array}{c}\text { CPU Freq. } \\
(\mathbf{M H z})\end{array}$ & $\begin{array}{c}\text { CPU Power } \\
(\mathbf{m W})\end{array}$ & $\begin{array}{c}\text { Latency } \\
(\mathbf{m s})\end{array}$ & $\begin{array}{c}\text { Performance } \\
(\mathbf{G F L O P} / \mathbf{s})\end{array}$ & $\begin{array}{c}\text { Energy } \\
(\mathbf{m J})\end{array}$ & $\begin{array}{c}\text { Energy Efficiency } \\
\text { (GFLOP/s/W) }\end{array}$ \\
\hline \multirow{3}{*}{$\begin{array}{c}\text { FC } \\
\text { HRL }\end{array}$} & \multirow{2}{*}{ Denver CPU } & 345 & 1,465 & 6.0 & 1.5 & 8.8 & 1 \\
\cline { 3 - 8 } & \multirow{2}{*}{ Arm A57 CPU } & 2,035 & 1,598 & 7.7 & 1.6 & 15.9 & 1 \\
\cline { 3 - 8 } & & 2,035 & 345 & 21.1 & 0.4 & 8.0 & 1 \\
\hline \multirow{3}{*}{$\begin{array}{c}\text { LSTM } \\
\text { HRL }\end{array}$} & \multirow{2}{*}{ Denver CPU } & 345 & 1,340 & 5.6 & 1.7 & 7.2 & 1.3 \\
\cline { 3 - 8 } & \multirow{2}{*}{ Arm A57 CPU } & 2,035 & 2,070 & 7.9 & 0.2 & 13.1 & 0.1 \\
\cline { 3 - 8 } & & 2,035 & 1,303 & 5.7 & 0.3 & 16.3 & 0.1 \\
\hline
\end{tabular}

the FC-HRL and the LSTM-HRL models, respectively. The signal power for the FC and the LSTM HRL models is $15 \%$ and $18 \%$.

\subsection{NVIDIA Jetson TX2 Implementation Results}

The architecture is designed to be flexibly deployable for general-purpose devices, allowing the developed machine learning models to be implemented on computing machines ranging from front-end edge devices to back-end computer servers. At least two hardware-level characteristics are assigned to all deep neural network models: model size and amount of computing operations per inference, all of which are upper-bounded by the platform resources they deploy to, or by the inference deadline. Both the hardware resource constraints and the diagnostic delay can satisfy the implementation targets while adding all the components of the framework together.

The trained models obtained from the previous section are deployed on two mobile CPUs including Denver (dual-core) and ARM-Cortex A57 (quad-core) implementation with different frequency settings. The NVIDIA Jetson TX2 development board, which has precise onboard power calculation, was used to perform all of the settings. We evaluated two different configurations, one with dual-core Denver CPU and another with quad-core ARM CPU. Table 4 shows the real-time implementation results in terms of latency, power, energy consumption, and energy efficiency for different models. Latency was minimum $10 \mathrm{~ms}$ for both the CPU-alone configurations when they were running at their maximum clock frequency of 2,035 MHz. The most energy efficiency was achieved when quad-core Arm CPU was running alone at its maximum frequency, which is $1.3 \mathrm{GFLOP} / \mathrm{s} / \mathrm{W}$ when only fully connected layers were used in the subgoal module and $0.3 \mathrm{GFLOP} / \mathrm{s} / \mathrm{W}$ when LSTM layers were used along with fully connected layers in the subgoal module. However, as the model is too small, the CPUs are underutilized. Moreover, small models do not benefit from heterogeneous platform such as GPU, therefore, GPU acceleration is not helpful for the proposed models.

\subsection{Discussion}

In recent years, many FPGA-based accelerators for convolutional neural network and computer vision applications have been proposed. This article proposed a scalable hardware for Reinforcement Learning applications that takes images as its input. Therefore, we compared our design with previous works that targeted computer vision and image classification applications. Implementation results show that compared to References $[10,39,44]$, our hardware with an optimum selection of configuration parameters, number of PEs, and frequency, consumes less energy per classification and is more energy-efficient. Compared to Reference [10], although our image dataset has a larger dimension, the results show significantly higher throughput and less power consumption and better energy efficiency. Additionally, our design shows a performance almost equivalent to 
Table 5. Comparison of the E2HRL Hardware Implementation Results with Similar Existing Works

\begin{tabular}{|c|c|c|c|c|c|c|c|}
\hline & [10] & [13] & [39] & [44] & [41] & FC-HRL & LSTM-HRL \\
\hline Application & $\begin{array}{c}\text { Image } \\
\text { Classification } \\
\text { (CIFAR) }\end{array}$ & $\begin{array}{c}\text { Physical } \\
\text { Activity } \\
\text { Monitoring }\end{array}$ & $\begin{array}{c}\text { Image } \\
\text { Classification } \\
\text { (CIFAR) }\end{array}$ & $\begin{array}{c}\text { Image } \\
\text { Classification } \\
\text { (CIFAR) }\end{array}$ & RL & RL & RL \\
\hline Platform & $\begin{array}{l}\text { FPGA } \\
\text { Zynq }\end{array}$ & $\begin{array}{l}\text { FPGA } \\
\text { Artix7 }\end{array}$ & $\begin{array}{l}\text { FPGA } \\
\text { Zynq }\end{array}$ & $\begin{array}{l}\text { FPGA } \\
\text { Zynq }\end{array}$ & $\begin{array}{l}\text { FPGA } \\
\text { Zynq } \\
\end{array}$ & $\begin{array}{l}\text { FPGA } \\
\text { Artix7 }\end{array}$ & $\begin{array}{l}\text { FPGA } \\
\text { Artix7 }\end{array}$ \\
\hline Input Dimension & $32 \times 32 \times 3$ & $64 \times 40 \times 1$ & $32 \times 32 \times 3$ & $32 \times 32 \times 3$ & $5 \times 1$ & $40 \times 30 \times 3$ & $40 \times 30 \times 3$ \\
\hline Precision & 16-bit & 16-bit & 16-bit & 32-bit & 32-bit & 32-bit & 32-bit \\
\hline Freq $(\mathrm{MHz})$ & 180 & 100 & 100 & 100 & 125 & 100 & 100 \\
\hline Throughput (fps) & 35.7 & 491 & 46.7 & 63.5 & 18.8 & 1,110 & 435 \\
\hline Perf (GOPS) & 0.94 & 1.6 & 1.23 & 1.67 & NR & 4 & 1 \\
\hline Power $(\mathrm{mW})$ & $>17,587$ & 175 & 2,944 & 1,015 & NR & 348 & 389 \\
\hline Energy (mJ/frame) & $>500$ & 0.35 & 63 & 33.7 & NR & 0.3 & 0.8 \\
\hline $\begin{array}{c}\text { Energy Efficiency } \\
(\text { GOPS/W) }\end{array}$ & $<0.05$ & 9.14 & 0.42 & 1.65 & NR & 11.4 & 3 \\
\hline
\end{tabular}

Reference [13] peak performance, despite using significantly larger input data. Comparison of this work with the existing FPGA-based deep neural network is summarized in Table 5.

As shown in Table 3, the best configuration in terms of energy efficiency is 8 PEs that resulted in an average of 11.4 and $3 \mathrm{GOP} / \mathrm{S} / \mathrm{W}$ energy efficiency and 0.3 and $0.8 \mathrm{~mJ}$ of energy for each inference for FC-HRL and LSTM HRL models, respectively. Low-power configurations consume 295 and $336 \mathrm{~mW}$ power to generate actions, which is suitable for embedded applications that usually have limited power envelope. Taking real-time vision application constraint into account [42], all of the configurations meet the typical 30 FPS constraint and consume less than $350 \mathrm{~mW}$.

\section{CONCLUSION}

In this article, we proposed E2HRL, a cross-layer energy-efficient hardware architecture design method using hierarchical Reinforcement Learning. We evaluate the performance of the model in several RL environments with different levels of complexity. Our Fully Connected and LSTM-based models show better performance compared to conventional RL, especially when the environment gets more complex. After a systematic model architecture optimization, the best configuration is selected to be implemented on hardware. We designed a scalable hardware architecture that is configurable with different number of PEs and could be implemented to achieve high throughput or low power consumption. The best configuration in terms of energy efficiency is $8 \mathrm{PEs}$, which resulted in an average of 11.4 and $3 \mathrm{GOP} / \mathrm{S} / \mathrm{W}$ energy efficiency and 0.3 and $0.8 \mathrm{~mJ}$ of energy for each inference for FC-HRL and LSTM HRL models, respectively. E2HRL low-power configurations consume 295 and $336 \mathrm{~mW}$ power to generate each action in the environment.

\section{REFERENCES}

[1] NVIDIA. 2020. NVIDIA jetson TX2. Retrieved from https://www.nvidia.com/en-us/autonomous-machines/embeddedsystems/jetson-tx2/.

[2] Jacob Andreas, Dan Klein, and Sergey Levine. 2017. Modular multitask reinforcement learning with policy sketches. In Proceedings of the International Conference on Machine Learning. PMLR, 166-175.

[3] Pierre-Luc Bacon, Jean Harb, and Doina Precup. 2017. The option-critic architecture. In Proceedings of the AAAI Conference on Artificial Intelligence, Vol. 31.

[4] Maxime Chevalier-Boisvert. 2018. Gym-MiniWorld environment for OpenAI Gym. Retrieved from https://github.com/ maximecb/gym-miniworld.

[5] Hyungmin Cho, Pyeongseok Oh, Jiyoung Park, Wookeun Jung, and Jaejin Lee. 2019. FA3C: FPGA-accelerated deep reinforcement learning. In Proceedings of the 24th International Conference on Architectural Support for Programming Languages and Operating Systems. 499-513. 
[6] Lucileide M. D. Da Silva, Matheus F. Torquato, and Marcelo A. C. Fernandes. 2018. Parallel implementation of reinforcement learning Q-learning technique for FPGA. IEEE Access 7 (2018), 2782-2798.

[7] William J. Dally, Yatish Turakhia, and Song Han. 2020. Domain-specific hardware accelerators. Commun. ACM 63, 7 (June 2020), 48-57.

[8] Muhammad Mudassir Ejaz, Tong Boon Tang, and Cheng-Kai Lu. 2021. A fast learning approach for autonomous navigation using a deep reinforcement learning method. Electron. Lett. 57, 2 (2021), 50-53.

[9] Sunil Gandhi, Tim Oates, Tinoosh Mohsenin, and Nicholas R. Waytowich. 2019. Learning behaviors from a single video demonstration using human feedback. In Proceedings of the 18th International Conference on Autonomous Agents and MultiAgent Systems. 1970-1972.

[10] Gopalakrishna Hegde, Nachiappan Ramasamy, Nachiket Kapre, et al. 2016. CaffePresso: An optimized library for deep learning on embedded accelerator-based platforms. In Proceedings of the International Conference on Compliers, Architectures, and Synthesis of Embedded Systems (CASES). IEEE, 1-10.

[11] Sepp Hochreiter and Jürgen Schmidhuber. 1997. Long short-term memory. Neural Computat. 9, 8 (1997), $1735-1780$.

[12] Morteza Hosseini, Mohammad Ebrahimabadi, Arnab Neelim Mazumder, Houman Homayoun, and Tinoosh Mohsenin. 2021. A fast method to fine-tune neural networks for the least energy consumption on FPGAs. UMBC Stud. Collect. (2021).

[13] Ali Jafari, Ashwinkumar Ganesan, Chetan Sai Kumar Thalisetty, Varun Sivasubramanian, Tim Oates, and Tinoosh Mohsenin. 2018. SensorNet: A scalable and low-power deep convolutional neural network for multimodal data classification. IEEE Trans. Circ. Syst. I: Reg. Pap. 99 (2018), 1-14.

[14] Mohit Khatwani et al. 2019. A low complexity automated multi-channel EEG artifact detection using EEGNet. In Proceedings of the IEEE EMBS Conference on Neural Engineering. IEEE.

[15] Changhyeon Kim, Sanghoon Kang, Sungpill Choi, Dongjoo Shin, Youngwoo Kim, and Hoi-Jun Yoo. 2019. An energyefficient deep reinforcement learning accelerator with transposable PE array and experience compression. IEEE Solidstate Circ. Lett. 2, 11 (2019), 228-231.

[16] Changhyeon Kim, Sanghoon Kang, Dongjoo Shin, Sungpill Choi, Youngwoo Kim, and Hoi-Jun Yoo. 2019. A 2.1 TFLOPS/W mobile deep RL accelerator with transposable PE array and experience compression. In Proceedings of the IEEE International Solid-State Circuits Conference (ISSCC). IEEE, 136-138.

[17] Nate Kohl and Peter Stone. 2004. Policy gradient reinforcement learning for fast quadrupedal locomotion. In Proceedings of the IEEE International Conference on Robotics and Automation. IEEE, 2619-2624.

[18] Amey Kulkarni. 2017. Heterogeneous and Scalable Sketch-based Framework for Big Data Acceleration on Low Power Embedded Cores. PhD Dissertation. University of Maryland, Baltimore County.

[19] Sicheng Li et al. 2015. FPGA acceleration of recurrent neural network based language model. In Proceedings of the IEEE 23rd Annual International Symposium on Field-Programmable Custom Computing Machines. IEEE, 111-118.

[20] Shanshan Liu, Xiaochen Tang, Farzad Niknia, Pedro Reviriego, Weiqiang Liu, Ahmed Louri, and Fabrizio Lombardi. 2021. Stochastic dividers for low latency neural networks. IEEE Trans. Circ. Syst. I: Reg. Pap. 68, 10 (2021), 4102-4115.

[21] N. K. Manjunath, A. Shiri, M. Hosseini, B. Prakash, N. R. Waytowich, and T. Mohsenin. 2021. An energy efficient EdgeAI autoencoder accelerator for reinforcement learning. IEEE Open f. Circ. Syst. 2 (2021), 182-195. DOI : https: //doi.org/10.1109/OJCAS.2020.3043737

[22] Volodymyr Mnih, Koray Kavukcuoglu, David Silver, Andrei A. Rusu, Joel Veness, Marc G. Bellemare, Alex Graves, Martin Riedmiller, Andreas K. Fidjeland, Georg Ostrovski, et al. 2015. Human-level control through deep reinforcement learning. Nature 518, 7540 (2015), 529.

[23] Andrew Y. Ng et al. 2006. Autonomous inverted helicopter flight via reinforcement learning. In Experimental Robotics IX. Springer, 363-372.

[24] Bharat Prakash et al. 2020. Guiding safe reinforcement learning policies using structured language constraints. In Proceedings of the 34th AAAI Conference on Artificial Intelligence: SafeAI Workshop. AAAI.

[25] Pedro Reviriego, Shanshan Liu, Otmar Ertl, Farzad Niknia, and Fabrizio Lombardi. 2021. Computing the similarity estimate using approximate memory. IEEE Trans. Emerg. Topics Comput. (2021).

[26] Andrei A. Rusu et al. 2017. Sim-to-real robot learning from pixels with progressive nets. In Proceedings of the Conference on Robot Learning. 262-270.

[27] John Schulman, Filip Wolski, Prafulla Dhariwal, Alec Radford, and Oleg Klimov. 2017. Proximal policy optimization algorithms. arXiv preprint arXiv:1707.06347 (2017).

[28] Masoud Shahshahani and Dinesh Bhatia. 2021. Resource and performance estimation for CNN models using machine learning. In Proceedings of the IEEE Computer Society Annual Symposium on VLSI (ISVLSI). IEEE, 43-48.

[29] Masoud Shahshahani, Mohammad Sabri, Bahareh Khabbazan, and Dinesh Bhatia. 2021. An automated tool for implementing deep neural networks on FPGA. In Proceedings of the 34th International Conference on VLSI Design and 20th International Conference on Embedded Systems (VLSID). IEEE, 322-327. 
[30] Shengjia Shao, Jason Tsai, Michal Mysior, Wayne Luk, Thomas Chau, Alexander Warren, and Ben Jeppesen. 2018. Towards hardware accelerated reinforcement learning for application-specific robotic control. In Proceedings of the IEEE 29th International Conference on Application-specific Systems, Architectures and Processors (ASAP). IEEE, 1-8.

[31] Aidin Shiri, Arnab Neelim Mazumder, Bharat Prakash, Houman Homayoun, Nicholas R. Waytowich, and Tinoosh Mohsenin. 2021. A hardware accelerator for language guided reinforcement learning. IEEE Des. Test (2021).

[32] Aidin Shiri, Arnab Neelim Mazumder, Bharat Prakash, Nitheesh Kumar Manjunath, Houman Homayoun, Avesta Sasan, Nicholas R. Waytowich, and Tinoosh Mohsenin. 2020. Energy-efficient hardware for language guided reinforcement learning. In Proceedings of the Great Lakes Symposium on VLSI. 131-136.

[33] David Silver et al. 2017. Mastering the game of Go without human knowledge. Nature 550, 7676 (2017), 354-359.

[34] Satinder Singh et al. 2002. Optimizing dialogue management with reinforcement learning: Experiments with the NJFun system. F. Arti. Intell. Res. 16 (2002), 105-133.

[35] Jiang Su, Jianxiong Liu, David B. Thomas, and Peter Y. K. Cheung. 2017. Neural network based reinforcement learning acceleration on FPGA platforms. ACM SIGARCH Comput. Archit. News 44, 4 (2017), 68-73.

[36] Richard S. Sutton, Andrew G. Barto, et al. 1998. Introduction to Reinforcement Learning, Vol. 2. The MIT Press, Cambridge, MA.

[37] Mineto Tsukada, Masaaki Kondo, and Hiroki Matsutani. 2020. A neural network-based on-device learning anomaly detector for edge devices. IEEE Trans. Comput. 69, 7 (2020), 1027-1044.

[38] Eric Tzeng et al. 2020. Adapting deep visuomotor representations with weak pairwise constraints. In Algorithmic Foundations of Robotics XII. Springer, 688-703.

[39] Ying Wang, Jie Xu, Yinhe Han, Huawei Li, and Xiaowei Li. 2016. DeepBurning: Automatic generation of FPGA-based learning accelerators for the neural network family. In Proceedings of the 53rd Annual Design Automation Conference (DAC'16). Association for Computing Machinery, New York, NY. DOI : https://doi.org/10.1145/2897937.2898003

[40] Hirohisa Watanabe, Mineto Tsukada, and Hiroki Matsutani. 2020. An FPGA-based on-device reinforcement learning approach using online sequential learning. arXiv preprint arXiv:2005.04646 (2020).

[41] Hirohisa Watanabe, Mineto Tsukada, and Hiroki Matsutani. 2021. An FPGA-based on-device reinforcement learning approach using online sequential learning. In Proceedings of the IEEE International Parallel and Distributed Processing Symposium Workshops (IPDPSW). IEEE, 96-103.

[42] Ming Yang, Shige Wang, Joshua Bakita, Thanh Vu, F. Donelson Smith, James H. Anderson, and Jan-Michael Frahm. 2019. Re-thinking CNN frameworks for time-sensitive autonomous-driving applications: Addressing an industrial challenge. In Proceedings of the IEEE Real-Time and Embedded Technology and Applications Symposium (RTAS). IEEE, 305-317.

[43] Chen Zhang et al. 2015. Optimizing FPGA-based accelerator design for deep convolutional neural networks. In Proceedings of the ACM/SIGDA International Symposium on Field-Programmable Gate Arrays. 161-170.

[44] Guanwen Zhong, Akshat Dubey, Cheng Tan, and Tulika Mitra. 2019. Synergy: An HW/SW framework for high throughput CNNs on embedded heterogeneous SoC. ACM Trans. Embed. Comput. Syst. 18, 2 (Mar. 2019). DOI : https: //doi.org/10.1145/3301278

[45] Yuke Zhu et al. 2017. Target-driven visual navigation in indoor scenes using deep reinforcement learning. In Proceedings of the IEEE International Conference on Robotics and Automation (ICRA). IEEE, 3357-3364.

Received June 2021; revised October 2021; accepted November 2021 